\title{
La forza del silenzio. \\ Dalla Filomela di Ovidio a Lisario di Antonella Cilento
}

\author{
The Power of Silence. \\ From Ovid's Philomela to Lisario by Antonella Cilento
}

\author{
Gerardina Antelmi \\ Università Adam Mickiewicz di Poznań \\ antelmi.g@gmail.com
}

\begin{abstract}
The present article examines how Antonella Cilento reinvents the myth of Philomela in Lisario o il piacere infinito delle donne, Premio Strega finalist (2014) and winner of Boccaccio Prize (2014). After evidencing the narrative aspects of Ovid's myth - the rape; the cutting out of the tongue depriving the raped woman of her voice; the expression of the violence she experienced - the analysis focuses on how Cilento overturns the myth by interweaving Ovid's tale with the topos of Sleeping Beauty. Despite her silencing, Lisario discovers and appropriates the pleasure that can be drawn from her body. The paper concludes by considering how the protagonist finds new and non violent ways to express herself and relate to future generations of women.
\end{abstract}

Keywords: Antonella Cilento, Philomela, contemporary Italian women's writing, women's voice

"Who peynted the leon, tel me who?" (The Wife of Bath's Tale) $)^{1}$

"Chi ha dipinto il leone?", chiede un leone di fronte a una rappresentazione in cui un contadino uccide appunto un suo simile. Se lo avesse dipinto un leone l'immagine sarebbe diversa. Così narra Alice di Bath, pellegrina dei Canterbury Tales chauceriani, la quale, proseguendo con l'analogia, sostiene che, se le donne

\footnotetext{
${ }^{1}$ The Wife of Bath, v. 688, Canterbury Tales, The Riverside Chaucer, L. Benson (Ed.), Oxford University Press, Oxford 1987.
} 
avessero scritto le storie, avrebbero raccontato degli uomini più malvagità di quanto il sesso maschile potrebbe rimediare. È proprio sulla base della 'differenza del punto di vista' che la presente analisi del romanzo Lisario o il piacere infinito delle donne di Antonella Cilento viene strutturata.

Lisario si presenta come un romanzo storico ambientato nella Napoli del XVII secolo in cui si muovono i pittori più famosi che da tutta Europa vi giungevano ${ }^{2}$ e che non solo interagiscono con la vita dei personaggi romanzeschi, Lisario, Avicente Iguelmano e Jacques Colmar, ma ne influenzano persino il destino - in primis de Sweerts che cerca la morte di Lisario per conquistare l'amore di Jacques. La narrazione procede al ritmo del romanzo picaresco, con alcuni riferimenti intertestuali espliciti a Cervantes - che la stessa Lisario legge riconoscendosi nella situazione di una delle sue protagoniste - e altri a Dante, Boccaccio fino a Shakespeare incastonati nella narrazione in un gioco di ammiccamento a chi legge che assume anche il carattere postmoderno del citazionismo nonché dell'ironia intertestuale.

Sebbene il romanzo sia una rilettura della fiaba della bella addormentata Lisario infatti si addormenta a piacere per rifiutare una situazione spiacevole impostale, quale ad esempio la possibilità di essere data in moglie a un vecchio "vavùso" e "zezzùso" (12) - si analizzerà Lisario dalla prospettiva della reinterpretazione del mito greco di Filomela, come tramandato da Ovidio. Dei numerosi fili che si intrecciano nel complesso arazzo della narrazione di Lisario per motivi di spazio se ne individuano qui tre che rimandano al mito di Filomela: la mutilazione che priva la donna della voce e la conseguente scrittura/tessitura segreta della propria esperienza; il matrimonio; infine il ritrovamento della voce tramite il canto dell'usignolo nel mito e di Teodora, la figlia di Lisario e Jacques, nel romanzo.

Secondo la versione delle Metamorfosi di Ovidio, Procne viene data in sposa dal padre Pandione a Tereo, re della Tracia, a suggellare la pace fra i due popoli. Quando Procne sente nostalgia della sorella, chiede a Tereo di andare da suo padre e scortare la sorella Filomela da lei. Durante il viaggio di ritorno Tereo rapisce Filomela e la violenta. Quando Filomela dichiara al cognato che proclamerà a tutto il mondo l'accaduto e l'infamia subita - rifiutando di suicidarsi e di assumersi la vergogna e il disonore - Tereo, dopo averle tagliato la lingua, la rinchiude in un luogo remoto nella foresta. A Procne spiega poi l'assenza di Filomela con la sua morte durante il viaggio. Nel frattempo Filomela prigioniera trova il modo di tessere una tela in cui rivela l'accaduto, e la manda alla sorella, la quale capisce immediatamente il messaggio. Liberata la sorella, Procne imbandisce un banchetto in cui serve in pasto a Tereo il loro figlio Iti. Quando scopre la verità, Tereo insegue le sorelle ma gli dèi presi da compassione trasformano i tre personaggi in uccelli.

\footnotetext{
${ }^{2}$ Michael de Sweerts (1618-1664); Ribera detto Spagnoletto (1591-1652); Pieter van Laer (15991641) per citarne alcuni.
} 


\section{IL SILENZIO E LA VOCE SEGRETA}

Il tema della voce, o meglio della mancanza della voce femminile, emerge sin dall'inizio in Lisario; la protagonista, infatti, è già mutilata e si esprime tramite lettere alla "Madonna Suavissima". La strategia narrativa del romanzo è duplice. La voce narrante onniscente che dipana le vicende, le intenzioni e i pensieri più remoti dei personaggi che si muovono intorno alla protagonista viene accompagnata dalle lettere che Lisario scrive in prima persona. Pertanto la voce narrante talvolta si ritrae per lasciar posto alle lettere di Lisario che aprono e concludono il romanzo. Nella lettera iniziale Lisario rivela la causa della sua menomazione: malata sin dalla nascita di "straparola", la fanciulla ha un gozzo per curare il quale è stata sottoposta a un'operazione chirurgica. Rivela, inoltre, come la sua propensione innata fosse quella di cantare.

Infatti appena partorita, già cantavo, squillante come tromba $[\ldots]$ crescendo, il vizio non scompariva, anzi vorticoso cresceva perché o io cantavo o io parlavo, come speditissimo predicatore, cosa vietatissima alle Piccole Femmine - e alle Grandi - dicendo tutto quello che mi passava per la testa. Suavissima, mi informarono che la Femmina è nata per obbedire, tacere e soffrire. E, a conferma, ogni volta che io cantavo o parlavo, ricevevo schiaffi e schiaffoni. [...] io il canto lo ringoiai una, due, mille volte, ed ecco all'improvviso una grande palla in gola! E più mi dicevano di stare zitta, più mi si gonfiava delle parole che non potevo dire e delle canzoni che non potevo cantare! (Lisario, 10, corsivo aggiunto).

Alla giovane Lisario, che non ha ancora imparato che le donne sono tenute al silenzio, viene diagnosticata la 'malattia' di "straparola" che deve essere curata con l'operazione che il chirurgo sbaglierà e che priverà la giovane della voce. Pertanto la giovane protagonista imparerà a sue spese ciò che $\mathrm{P}$. Klindienst Jones definisce la "natura conflittuale della scoperta del linguaggio" $(2002,261)$. Lisario aggiunge in quale modo rocambolesco e miracoloso abbia appreso a leggere e scrivere: dopo essere stata colpita alla testa da un poderoso tomo mentre si aggirava nella biblioteca paterna, naturalmente di nascosto, ha perso i sensi. Quando si riprende non solo può decifrare quei simboli, ma li sa anche riprodurre. Decide, quindi, di conservare segreta la sua capacità di scrittura e di mantenere agli occhi di tutti l'apparenza di "gallina" mentre aveva acquisito "l'anima di volpe" (10).

È interessante notare che l'operazione chirurgica che inciderà sul destino di Lisario abbia per oggetto la gola. Secondo una "topografia del corpo" individuata da Nicole Loraux, la gola, la parte anteriore del collo, punto forte della bellezza femminile nella tradizione della tragedia greca, è al contempo un punto altamente vulnerabile. È il punto in cui la lama del sacerdote pagano rappresentato nella tragedia greca colpisce le giovani donne prescelte come vittime sacrificali (Loraux, 1991, 50). Le eroine greche muoiono per un colpo alla gola, che si tratti di sacrificio, suicidio 
o assassinio, mentre nell'epica i guerrieri muoiono per un colpo ricevuto al collo o per ferite al petto, da veri eroi (Loraux, 1991, 52, 60). La ripetizione di questo schema narrativo farebbe pensare a una spiegazione esterna al mondo della tragedia, secondo la quale il corpo della donna sarebbe considerato come definito fra due orifizi, fra due colli (Loraux, 1991, 61). Come osserva Cavarero (2010), "[c]iò che identifica il genere femminile, il suo proprium, è la parte del corpo sotto la cintola. La testa, tanto più come sede dell'intelligenza, non entra, di solito, fra le caratteristiche necessarie" (122).

La ferita alla gola infertale dal medico non provocherà la morte di Lisario, bensì la priverà della voce. Priva di parola, Lisario perde parte della sua umanità. Come per Filomela il taglio della lingua può essere interpretato come un secondo stupro (Marder, 1992, 158), o persino terzo se, come osserva Segal (1993), Filomela subisce già "una penetrazione aggressiva dello sguardo maschile" (260) di Tereo non appena la giovane gli appare. Può, inoltre, essere letto come perdita della qualità precipua dell'umanità in quanto viene distrutta la capacità di parlare (Segal, 1993, 267), che sin dai tempi di Aristotele è la capacità che differenzia l'essere umano dagli animali. Secondo Aristotele, infatti, l'essere umano è zoon logon echon, vale a dire un "vivente che ha logos", un "animale parlante" (Cavarero, 2010, 44). È la parola che distingue l'essere umano dagli animali poiché la sua voce è dotata di significato, mentre la voce degli animali è solo segno, di piacere o di dolore ma priva di significato (Cavarero, 2010, 44). Secondo la prospettiva maschile Lisario, donna giovane e muta, rappresenta l'ideale dell'oggetto del desiderio dell'uomo. La donna perfetta è la donna muta, ma in grado di ascoltare e quindi di obbedire, "la donna che ha perso la voce [...] che non può parlare" (Cavarero, 2010, 119, 130). Lisario, la fanciulla che non solo parlava ma che sin dalla nascita cantava, risulta quindi doppiamente privata della sua voce peculiare, se, come sostiene Cavarero (2010), "[n]essuna donna è appunto più muta di quella che perde la voce del canto" (132).

La voce di Lisario trova corpo in un modo di espressione tutto femminile, quello epistolare (Doglio, 2000, 13-24). Il fatto che le lettere siano rivolte alla Madonna conferisce alla voce di Lisario non solo la freschezza dell'ingenuità ma anche e principalmente una nota di sincerità come la si può trovare nell'atto della confessione, confermata appunto dalla scelta dell'interlocutrice divina. Alla Madonna, la divinità femminile che tutto vede e che mantiene al contempo la sua parte terrena, corporea, Lisario confiderà i suoi pensieri più reconditi. L'invocazione epistolare alla Madonna richiama altresì quell'invocazione alle Muse risalente alla tradizione classica: nella Madonna Lisario trova conforto, ispirazione e anche quel sostegno sovraumano, divino, che salverà lei e sua figlia Teodora. Grazie a una sua apparizione in sogno, Lisario si sveglia dalla sua ultima catalessi sfuggendo a morte sicura e potrà iniziare una nuova fase della sua vita con la figlia lontane dalla famiglia di origine e dal marito. 
Sin dall'esordio, pertanto, la voce dell'eroina si inserisce nel filone della re-visione del mito in cui la protagonista riesce, nonostante la mutilazione della voce, a esprimere la propria esperienza dalla prospettiva femminile. Si pensi non solo a Marianna Ucrìa di Dacia Maraini e a Storie di Patio di Fabrizia Ramondino, ma anche alla protagonista di Quaderno proibito di Alba de Cèspedes che inizia la scoperta della propria voce con una doppia trasgressione: l'acquisto di un quaderno in un giorno proibito nonché proprio il dedicarsi alla scrittura, che deve rimanere attività da tenere nascosta, di cui vergognarsi quasi ${ }^{3}$. In modo simile Lisario scriverà le lettere segrete che nasconde in una grotta protette dal mare. Per Lisario, tuttavia, la scrittura è "l'Arte del Diavolo", mentre il Canto che mi è impedito, corre verso il cielo e tocca le stelle..." (55, corsivo aggiunto). Per quanto riguarda il mito di Filomela, la riscrittura risulta fondamentale al fine di riequilibrarne la ricezione poiché tale mito è ricordato soprattutto per il finale macabro, per la violenza delle donne (Joplin, 2002, 272) piuttosto che per la violenza di Tereo nei confronti di Filomela e per i tradimenti dello stesso Tereo nei confronti della consorte Procne, di Filomela, e del suocero e di conseguenza nei confronti del sistema patriarcale stesso, non avendo mantenuto la parola data a Pandione. Nella ricezione del mito del secolo XVI, ad esempio, le due sorelle erano considerate le responsabili per aver innescato la tragedia: Procne con il suo desiderio espresso di rivedere la sorella, e dopo lo stupro Filomela con la sua dichiarazione di denuncia nei confronti di Tereo - dichiarazione che viene considerata appunto causa della sua mutilazione (Trivellini, 2015, 94). Responsabilità nel caso di stupro che ancora oggi viene attribuita alla donna che subisce violenza anziché all'autore della violenza. La voce delle donne deve rimanere silente, insegnerebbe il mito interpretato dalla prospettiva maschile. D'altro canto, secondo la critica femminista la 'voce' viene intesa come acquisizione di una autorevolezza testuale, espressione dell'esperienza delle donne attraverso la scrittura; acquisizione di una 'voce' che evidenzi le qualità peculiari della esperienza culturale delle donne con una 'voce differente' (Dunn, Jones, 1994, 1). Benché mutilata, Filomela riesce a narrare comunque la sua storia; e nel mito ovidiano gli dèi mostrano benevolenza nei confronti delle donne che vengono tramutate in uccelli, in particolare in usignolo ${ }^{5}$. L'associazione della voce sia con il textum

\footnotetext{
${ }^{3}$ In particolare di Fabrizia Ramondino si veda il racconto 'La Signora di Son Batle' in cui la narratrice che ha subito violenza si esprime attraverso la tessitura. Cfr. A. Giorgio (1993), 'Narrative as Verbal Performance: Énonciation and Énoncé in Fabrizia Ramondino's "La signora di Son Batle", Italian Studies, 48, 86-106. La protagonista del romanzo di de Cèspedes sente l'urgenza di comprare un quaderno, un articolo che non potrebbe essere venduto di domenica dal tabaccaio secondo una legge dell'epoca.

${ }^{4}$ Lisario spiega che la scrittura è l'arte del Diavolo perché si formano i segni della scrittura "a volte anche con il ventre poggiato a terra, di nascosto e a lume di moccolo, che così mi vengono strofinamenti e pensieri..." (55).

${ }^{5}$ Per quanto riguarda lo sviluppo dell'associazione dell'usignolo con la poesia nella storia letteraria europea. Cfr. J. Williams (1997), Interpreting Nightingales. Gender, Class and Histories, Sheffield: Sheffield Academic Press.
} 
- della tessitura e della parola scritta - sia con il canto percorre il mito e Lisario. È una voce quella di Lisario genuina, fresca e senza malizia. E che spesso fa sorridere. Per esempio quando riflettendo sul matrimonio si chiede e chiede alla Madonna scusandosi - se rispetto a San Giuseppe, vecchio e stanco, l'angelo biondo del Signore non fosse più prestante (54). Sul matrimonio Lisario ritornerà spesso a riflettere. È quindi opportuno esaminare questo aspetto in modo più dettagliato.

\section{IL MATRIMONIO}

Lisario è riuscita a evitare il matrimonio con il vecchio bavoso, cui si è accennato sopra, addormentandosi per mesi. Per inciso l'età del matrimonio coincide con il sopraggiungere delle mestruazioni e quindi con la capacità riproduttiva della donna. Lisario si sveglia ad opera di Avicente Iguelmano, un medico spagnolo con pessima reputazione professionale in fuga dal suo paese e dall'Aja. Avicente è diventato medico seguendo la volontà paterna benché il suo carattere non fosse adatto a tale professione, come dimostrerebbero i suoi svenimenti giovanili durante le lezioni di anatomia. Giunto a Napoli, come prima prova gli viene affidato il compito in cui tutti gli altri medici hanno fallito: svegliare Lisario. Vi riesce quando, dopo giorni di osservazione, decide di spogliarla e di esplorarle i genitali. Dopo qualche tempo, al risveglio di Lisario, il padre offre la figlia in moglie al medico. Quando apprende che la giovane è muta, ignaro dell'abilità di scrittura di Lisario, Iguelmano pensa che sia il "delitto perfetto" in quanto la donna non potrà mai rivelare il modo in cui lui l'ha 'guarita', violandola. Ben presto Lisario si allontana dal marito quando questi la violenta durante la vita matrimoniale, si distacca ulteriormente dal matrimonio e riflette nelle sue lettere alla Madonna sui suoi doveri e su cosa sia il matrimonio: "Lo so ora Tu mi condannerai e così il Mondo, ma che ci posso fare? Ho forse giurato al prete di sopportare Schifo, Disgrazia e Fastidio? Non mi sembrava che la formula recitasse vessazioni!" (160). Nel frattempo avviene l'incontro fra Lisario e Jacques, uno scenografo ebreo francese, con cui avrà la figlia Teodora. Dopo una breve fuga e convivenza con Jacques, Lisario viene riportata con la figlia in casa dei genitori per vivere di nuovo con il marito. Apparentemente l'ordine viene ristabilito, come desiderava Iguelmano, per salvaguardare il suo onore maschile, ma per poco perché Lisario si addormenta di nuovo.

Il matrimonio rappresenta l'istituzione fondamentale su cui si basa il sistema patriarcale, in cui Lisario viene data dal padre ad Avicente con lo scopo di diventare moglie e procreatrice di stirpe, qualunque sia la volontà di Lisario in proposito. Come osserva Irigaray (1985), la donna non può essere in controllo della sua relazione con la maternità, in quanto non c'è differenza fra essere donna ed essere madre (143). Per il medico, il matrimonio rappresenta l'inizio di una brillante carriera coronata da potere, autorità, reputazione professionale e denaro. Tuttavia in Lisario 
il matrimonio viene posto in contrasto al rapporto consapevole, libero e paritario fra donna e uomo, esemplificato dal rapporto di Lisario con Jacques, che non è matrimonio bensì convivenza. Come nel mito il matrimonio ha la funzione di suggellare la pace fra due popoli tramite la cessione di una figlia come moglie di Tereo, così per Lisario il matrimonio è il risultato di un patto fra uomini. In questo ordine sociale, le donne sono 'merci', e in quanto tali non possono avere il diritto di parlare durante lo scambio fra uomini (Irigaray, 1985, 84). Secondo quanto affermava Lévi-Strauss a proposito dell'istituzione del matrimonio, "la riduzione dell'altro sesso a merce di scambio, per quanto ingiusto, avrebbe reso possibile il passaggio dalla bestia all'uomo, cioè l'evoluzione dalla stato selvaggio alla civiltà" (De Carneri, 2015, 69). Tramite lo scambio delle donne fra gruppi di uomini si eviterebbe l'incesto (Irigaray, 1985, 170) ${ }^{6}$. Inoltre, gli uomini si scambierebbero le donne nello stesso modo in cui si scambiano parole: una donna avrebbe significato come la figlia di un padre, la sorella di un fratello, la moglie di un marito (De Carneri, 2015, 70). Interessante, quindi, osservare il collegamento fra matrimonio, linguaggio e mutilazione femminile che conduce la donna al silenzio.

Lo scambio delle donne avviene in stretta relazione al controllo del corpo femminile da parte degli uomini, sia prima del matrimonio da parte del padre, sia durante il matrimonio da parte del marito. Vale la pena ricordare qui il parallelismo posto fra corpo sociale e corpo fisico inteso come una immagine della società. Interesse e difesa delle entrate e uscite della città, difesa dalle invasioni sono riflessi nella difesa dei confini del corpo fisico, in particolare quello femminile, con una gerarchia che include il capo e i piedi, gli organi sessuali e gli orifizi (Douglas, 1996, 74). Parallelamente e in contrasto a tale gerarchia, il disordine sociale viene espresso con immagini di impurità e pericolo (Douglas, 1996, 86). Risulta particolarmente significativo come nel romanzo proprio quando esplode la rivolta popolare guidata da Masaniello, un evento che manda "per aria tante convenzioni" (194), Lisario per la prima volta nella sua vita non dorme nella casa paterna. La giovane si è salvata dal 'disordine' della rivolta rifugiandosi nella bottega di un tipografo dove ha trovato un foglio della Bibbia su cui scrive alla Madonna. Vedendo il risultato della rivolta popolare - lussuose carrozze trainate da buoi, donne lerce con perle al collo - conclude che "il mondo è alla rovescia" (201). Si rende, tuttavia, conto di non essere "mai stata nel mondo e che non sapev[a] cosa fosse, sola come non er[a] mai stata" (203). In questo momento di capovolgimento dell'ordine sociale convenzionale, Lisario e Jacques si ritrovano e decidono di convivere. È proprio in questo momento di mancanza di controllo dei confini del corpo sociale e fisico - non solo della protagonista - che Lisario e Jacques iniziano la loro convivenza scelta e cele-

\footnotetext{
${ }^{6}$ Entrambe le studiose fanno riferimento alle opere di Claude Lévi-Strauss, Irigaray a The Elementary Structures of Kinship, trans. J. Harle Bell, J. Richard von Sturner, R. Needham (Boston 1969), p. 36; Carneri a Linguaggio e parentela, in Id. Antropologia culturale, Il Saggiatore, Milano 2002.
} 
brata in opposizione all'istituzione del matrimonio: si dichiarano 'sposati' in modo indipendente e in assenza di rappresentanti dello stato e della religione a testimoni. In contrasto all'istituto del matrimonio, come si è accennato, Lisario vive nel rapporto con Jacques, un rapporto consapevole e paritario fra due individui. Lisario e Jacques convivono, fuori dal sistema del riconoscimento dell'autorità maschile, in cui entrambi gli individui, donna e uomo, affermano la propria volontà.

Fuori è salita la notte e Jacques mi ha detto "Adesso io e te siamo sposati. E a nessuno dobbiamo più rendere conto. [...] quasi non sento la mancanza dei miei Libri: ho potuto cucire questo foglio a un nuovo quaderno, segno chiaro di Nuova Vita e Nuovo Tempo!" (203, corsivo aggiunto).

E più avanti Lisario spiega il motivo del suo amore per Jacques: "Amo Jacques perché ha capito che non deve trattarmi come una sposa qualunque, altrimenti di nuovo dormirei!" (232).

\section{LA NUOVA VOCE - IL CANTO}

Una volta scoperte le lettere alla Madonna e apprendendo così il tradimento della moglie, Avicente decide di vendicarsi. Non solo e non tanto del tradimento, quanto perché come uomo non può accettare che Lisario "pensasse e con il suo pensiero lo giudicasse non temendolo o odiandolo [...] ma provando per lui disgusto, indifferenza e, peggio del peggio, ridicolo" (166). Tuttavia il pensiero della scrittura della moglie lo tormenta. È consapevole, infatti che "[a]nche se [il quaderno] spariva, Lisario avrebbe potuto riscrivere la sua storia, dunque era come minimo necessario amputarle le mani" (169). Sebbene Avicente non riesca a realizzare il suo proposito di amputazione, il suo intento non può non richiamare il fato di Lavinia del Tito Andronico di Shakespeare. Tale riferimento intertestuale esemplifica come, tramite la conoscenza della tradizione letteraria, l'uomo-padre-marito e come tale in controllo del corpo della donna, apprenda e si approprii dell'esperienza maschile precedente. Già nel Tito Andronico shakespeariano sia lo stupratore sia il padre di Lavinia, apprendendo dal mito ovidiano, evitano gli errori di Tereo: lo stupratore amputandole le mani e il padre, ritenendola comunque colpevole, la uccide. Nella sua posizione di stupratore ma soprattutto di uomo che è venuto meno al contratto fra uomini che gli garantisce la sua posizione di privilegio all'interno del sistema patriarcale, Avicente come Tereo deve tacitare la 'voce' della donna per mantenere reputazione e ricchezza. Come sottolinea Joplin, nel privato la forza sarebbe sufficiente a tacitare la donna; ma se la voce della donna venisse resa pubblica, la donna e l'uomo sarebbero "equal", avrebbero pari importanza $(2002,263)$. Non solo. Nella sua estenuante ricerca dell'origine del piacere sessuale femminile Avicente percepisce il potere 'indicibile' della capacità di godere delle donne. Ne capta, se pur 
labilmente, il potere che potrebbe scaturirne: un potere che rischierebbe di incrinare e far crollare il sistema di potere patriarcale. Sebbene la sua ricerca sul piacere femminile - iniziata dopo la guarigione di Lisario - rimanga incompresa dagli altri personaggi (quali ad esempio Tonno d'Agnolo che fornisce ad Avicente le prostitute per i suoi studi) e li faccia ammiccare per l'oggetto della sua ricerca, Avicente confessa i suoi timori a Tode, il notomista. Proprio nel laboratorio di quest'ultimo dove giacciono principalmente cadaveri di donne sezionate in cui l'uomo di scienza è alla ricerca della verità limitandosi all'esplorazione del corpo - Avicente esprime i suoi timori sul piacere che le donne provano poiché, ritiene, in questo modo agli uomini "rubano qualcosa" (177).

“[...] [le donne] se possono godere senza di noi, forse possono fare altro senza di noi [...] Immaginate che concepiscano senza di noi... In fondo Maria Vergine....”.

"Favole. Anche i preti sanno che è una menzogna".

"Ma fate conto che sia una metafora, fate conto che sia un mito che racconti un'altra verità $[. .$.$] Immaginate un mondo senza padri: su chi eserciteremmo la nostra... autorità?$ [...] Saremmo alla mercé di chi partorisce i figli e li educa... Presto anche il denaro sarebbe nelle loro mani” (177).

Benché Tode reiteri la concezione 'scientifica' dell'inferiorità della donna, Avicente non abbandona la sua intuizione, dal momento che la lettura delle pagine di Lisario lo porta a concludere che

[...] il segreto che aveva a lungo cercato fra le gambe della moglie era invece lì, nel suo quaderno, seppellito in quell'anima che non poteva parlare ma che a dispetto e all'insaputa di tutti, sapeva scrivere. Questo pensiero lo attraversò come una fitta e subito fu travolto dalla furia e dalla negazione, poiché, se non poteva concedere a Lisario di godere per suo conto, meno ancora poteva lasciare che pensasse e che con il suo pensiero lo giudicasse non temendolo o odiandolo, come si doveva a un marito inviso o a un padrone, ma provando per lui disgusto, indifferenza e, peggio del peggio, ridicolo (166).

Avicente intuisce che il "piacere femminile rappresenta una delle maggiori minacce a ogni discorso maschile, rappresenta la sua più irriducibile esteriorità o extraterritorialità" (Irigaray, 1985, 157, trad. mia). Proprio per questa raggiunta consapevolezza, oltre alla volontà di punirla per l'adulterio e "lavare col sangue l'onta subita, al riparo della legge" (167), Avicente tenta di cucire le grandi labbra di Lisario in modo che il figlio non nasca. Pensiero e tentativo di una valenza altamente simbolica se si considera, come si è evidenziato sopra, il significato degli orifizi del corpo fisico che assurgono a rappresentazione di corpo sociale. Inoltre il proposito di Avicente non solo si rifà all'atto di violenza della mutilazione mitologica ovidiana ma intende oltrepassare persino la lezione shakespeariana: tacitare tramite amputazione della lingua e delle mani non è sufficiente, è necessario impedire la creazione, 
l'espressione della donna, in qualunque forma essa si presenti, chiudendo ogni 'varco' di entrata o di uscita del corpo fisico-sociale della donna.

Ma grazie alla sua conoscenza letteraria anche Lisario apprende, leggendo dell'esperienza delle eroine letterarie principalmente il 'non detto' della prospettiva delle eroine stesse. Riflettendo sulla sua relazione con Jacques - quando ancora è considerata adulterio - e ricordando la vicenda di Desdemona e Otello, Lisario si chiede a cosa servano i libri "se non si impara cosa non fare?" (159). Iguelmano, tuttavia, non riesce a fare in modo che la comunicazione di Lisario sia interrotta definitivamente. Lisario non ha mai smesso di scrivere le sue lettere alla Madonna, e per chi legge, la protagonista non ha mai perso la voce. Le lettere che lei scrive, poste a conclusione delle varie sezioni narrative, le completano con il suo punto di vista, e fanno da filo conduttore del romanzo anche quando Lisario non compare.

Il timore di Iguelmano risulterà fondato: Lisario riscrive la sua storia. Come Filomela manda la tela alla sorella, così Lisario riesce a comunicare alla figlia la sua prospettiva della storia. Paradossalmente, la scrittura, la tessitura, la creazione di un textum in reclusione, proprio perché 'tagliata' fuori da influenze canoniche appartenenti alla tradizione maschile, fa sì che Lisario, come Filomela, si muova in una condizione intellettuale di libertà e indipendenza che permette loro di esprimersi non solo in modo sciolto da lacci di canone e stile ma anche con sincerità e mancanza di reticenza. In altri termini, trovano la loro 'voce' che altre donne comprendono. Come nota Ovidio, il fatto che Procne potesse comprendere il messaggio di Filomela "mirum fuisse" (v. 581), aveva del meraviglioso, dello straordinario.

Per concludere, una rilettura del mito in generale e del mito di Filomela in particolare risulta fruttuosa ancora oggi. Come nota Joplin, il telaio di Filomela ci parla ancora in quanto rappresenta la volontà di sopravvivenza malgrado tutto ciò che minaccia di ridurre la donna al silenzio, inclusa la tradizione letteraria maschile e i suoi critici $(2002,272)^{7}$. Anche in Lisario il tema della voce rimane centrale, come si è dimostrato fin qui. La possibilità di esprimere la propria 'voce' e di tramandare la propria prospettiva della storia si potrebbe interpretare in senso più ampio come la possibilità di mantenere intatto quel filo simbolico del discorso fra generazioni di donne, la possibilità di avere accesso a quegli scritti della storia femminile che sono rimasti occultati per secoli. In tal modo la figlia, Teodora, acquisisce conoscenza e conduce una vita diversa dalla madre. Superando la teoria della 'uccisione' della madre per parlare invece in termini di relazionalità con essa, è necessario "trovare, ritrovare, inventare le parole, le frasi che dicono il rapporto più arcaico e più attuale con il corpo della madre, con il nostro corpo, le frasi che

\footnotetext{
${ }^{7}$ Per quanto riguarda un'analisi ancora attuale sulla relazione fra il mito di Aracne e Filomela, la tessitura e la critica letteraria, si veda N.K. Miller (1986), 'Arachnologies: The Woman, the Text, and the Critic'. In N.K. Miller (Ed.), The Poetics of Gender (pp. 270-295), New York: Columbia University Press.
} 
traducono il legame con il suo corpo, il nostro, quello delle nostre figlie" (Irigaray, 1989, 30). Inoltre, Teodora può essere interpretata come una figlia anche simbolica, oltre che naturale. Lisario e Teodora comunicano. Teodora riesce a leggere la storia scritta dalla madre. Non solo. Ne eredita la voce ed è proprio sulla voce che si centra la professione di Teodora. Questo rapporto madre-figlia naturale potrebbe essere, a mio avviso, anche simbolico, un passaggio di testimone, per così dire, generazionale. Cilento, infatti, assimila la re-visione del mito e con esso il tema della voce dalle scrittrici delle generazioni precedenti, lo rielabora includendovi la testimonianza dell'eredità raccolta proprio tramite il personaggio della figlia Teodora con la sua professione di cantante. In tal modo la prospettiva cambia radicalmente rispetto ai romanzi citati poc'anzi. Basti qui pensare a Quaderno proibito che si conclude con la distruzione del diario da parte della protagonista, diario in cui la voce che scaturiva da quegli incontri intimi con se stessa risultava talvolta di disturbo alla protagonista stessa. Il romanzo di Maraini La lunga storia di Marianna Ucria sebbene termini con la protagonista che, sentendo "le alette ai piedi", decide di andare per il mondo, si conclude con la riflessione "[l]a risposta è muta". La narratrice del racconto di Ramondino decide di non denunciare a voce la violenza subita, di tesserla come Filomela ma di affidare il suo testo-tessuto a un uomo.

Nelle lettere Lisario riflette e ridescrive i termini di 'matrimonio', come una unione di anime che si materializza nella convivenza voluta da ambedue gli individui; riscrive i termini del concetto di 'amore', che non nasce dall'attrazione maschile verso la bellezza, in cui trova radici il desiderio di possesso dell'altro; riscrive l'idea della 'maternità' come scelta personale della donna piuttosto che destino biologico imposto dall'imperativo di procreare al fine della sopravvivenza della famiglia patriarcale. La lettura delle lettere di Lisario-madre dà consapevolezza e contribuisce a fornire la voce, in forma di canto, a Teodora, ormai divenuta cantante di professione. Sebbene si presenti in scena come castrato, si potrebbe leggere il successo della figlia come cantante lirica, con l'osservazione di Cavarero secondo cui "la cantante lirica diventa una figura straordinariamente potente [...] [che] anche svolge la funzione maschile attiva, di chi penetra e inonda" (143). Teodora, dunque, trova la voce che a Lisario era stata negata; realizza la speranza di Lisario espressa poco prima del parto: "Davvero vorrei indietro la mia voce per porre domande. Mi tocco la gola. So che le donne urlano durante il parto e io non potrò. Piangerà per me la creatura che nascerà" (229). La sorte di Lisario è riscattata da e tramite Teodora che nasce e mantiene la sua voce squillante quanto la voce che avrebbe avuto Lisario se non fosse stata mutilata. Consapevole della storia della madre, la figlia cantante diventa 'uccello' analogamente a Filomela alla conclusione del mito ovidiano.

Teodora non sta zitta un attimo e io sono vendicata: per lei parlano le migliaia di parole, di voci, di sentimenti che in vita mia ho dovuto tacere. Ha la gola che partorisce legioni di uccelli in volo. Canta le nuvole, canta i fiumi e le isole, canta la progenie delle specie 
di cui Tu e Dio Padre avete popolato il Mondo. Grazie, Suavissima... (275, corsivo aggiunto).

Infine, ci piace distaccarci di un passo dalla trama tessuta da Cilento in Lisario e soffermarci forse sul più significativo fra i numerosi riferimenti intertestuali presenti nell'opera: la scelta del nome della protagonista, presentata come "una lucente e verde lucertola" (30). Il suo nome si presta al gioco di parole "Lisario, Lézard, Lizard, Lucertola" (296), come la chiama Jacques e come l'ha ritratta: "una lucertola spaventata ma che non indietreggia" (296). Lisario costituisce sì riferimento all'iguana-Iguana di Anna Maria Ortese, (comunicazione personale di A. Cilento, 16 luglio 2016) ma soprattutto riconoscimento di un'altra voce di donna indiscussa fra le autrici del '900 italiano, una 'madre' - ispirazione letteraria con cui relazionarsi. $\mathrm{Ci}$ piace vedere in questa celebrazione intertestuale un filo di comunicazione fra generazioni di donne non solo romanzesche, come Teodora che 'legge' Lisario, ma anche e principalmente generazioni di donne reali, autrici che si ri-trovano, comunicano e rimangono in relazione.

Ringrazio le/i recensori per le loro preziose e puntuali osservazioni sulla prima stesura del presente articolo; ringrazio anche le colleghe di facoltà e le colleghe incontrate durante il convegno SIS 2015 per le preziose osservazioni e le studentesse con cui abbiamo discusso il romanzo Lisario durante i corsi. 


\section{BIBLIOGRAFIA}

Cavarero, A. (2010). A più voci. Filosofia dell'espressione vocale. Milano: Feltrinelli.

Cilento, A. (2014). Lisario o il piacere infinito delle donne. Milano: Mondadori.

De Carneri, M. (2015). Il fallo e la maschera. L'inconscio patriarcale della psicanalisi. Milano: Mimesis.

Doglio, M.L. (2000). Letter Writing, 1350-1650. In L. Panizza, S. Wood (Eds.), A History of Women's Writing in Italy (pp. 13-24). Cambridge: Cambridge University Press.

Douglas, M. (1996). Natural Symbols. Exploration in Cosmology. London: Routledge.

Dunn, L., Jones, N. (1994). Embodied Voices: Representing Female Vocality in Western Culture. Cambridge: Cambridge University Press.

Giorgio, A. (1993). Narrative as Verbal Performance: Énonciation and Énoncé in Fabrizia Ramondino's "La signora di Son Batle". Italian Studies, 48, 86-106.

Irigaray, L. (1985). This Sex Which is Not One. New York: Cornell University Press.

Irigaray, L. (1989). Sessi e genealogie, trad. L. Muraro. Milano: La Tartaruga.

Joplin Klindienst, P. (2002). The Voice of the Shuttle Is Ours. In L.K. McClure (Ed.), Sexuality and Gender in the Classical World (pp. 259-86). Oxford: Blackwell Publisher.

Lévi-Strauss, C. (1969). The Elementary Structures of Kinship, trad. J. Harle Bell, J.R. von Sturner, R. Needham. Boston: Beacon Press.

Loraux, N. (1991). Tragic Ways of Killing a Woman, trad. A. Forster. Cambridge Mass.: Harvard University Press.

Marder, E. (1992). Disarticulated Voices: Feminism and Philomela. Hypatia, 7, 148-166.

Miller, N.K. (1986). Arachnologies: The Woman, the Text, and the Critic. In N.K. Miller (Ed.), The Poetics of Gender (pp. 270-295). New York: Columbia University Press.

Ovidio, P. (2015). Metamorfosi. Torino: Einaudi.

Segal, C. (1993). Philomela's Web and the Pleasures of the Text: Reader and Violence in the Metamorphoses of Ovid. In I.J.F. de Jong, P. Sullivan (Eds.), Modern Critical Theory and Classical Literature (pp. 257-280). New York: Leiden.

Trivellini, S. (2015). The Myth of Philomela from Margaret Atwood to... Chaucer: Contexts and Theoretical Perspectives. Interférences littéraires, 17, 85-99.

Williams, J. (1997). Interpreting Nightingales. Gender, Class and Histories. Sheffield: Sheffield Academic Press. 